\title{
RECIPROCAL SYSTEMS OF LINEAR DIFFERENTIAL EQUATIONS*
}

BY

\section{E. J. WILCZYNSKI}

\section{§1. Dualistic geometrical interpretations of a system of two linear differential equations of the second order.}

The geometrical interpretation adopted in a former paper $\dagger$ was as follows. The system

$$
\begin{aligned}
& y^{\prime \prime}+p_{11} y^{\prime}+p_{12} z^{\prime}+q_{11} y+q_{12} z=0 \\
& z^{\prime \prime}+p_{21} y^{\prime}+p_{22} z^{\prime}+q_{21} y+q_{22} z=0
\end{aligned}
$$

with the independent variable $x$ was given. Its integration made known four pairs of independent simultaneous solutions

$$
y_{k}=f_{k}(x), \quad z_{k}=g_{k}(x) \quad(k=1,2,3,4)
$$

The quantities $\left(y_{1}, y_{2}, y_{3}, y_{4}\right)$ and $\left(z_{1}, z_{2}, z_{3}, z_{4}\right)$ were interpreted as the homogeneous coördinates of two points $P_{y}$ and $P_{z}$, so that equations (2) represented two curves in space, $C_{y}$ and $C_{x}$. Corresponding points of the two curves $C_{y}$ and $C_{z}, \imath$. e., points belonging to the same value of $x$, were joined by a straight line $L_{y z}$. All of these straight lines generated a ruled surface $S$ which was never a developable surface. The surface $S$ was the same for all systems equivalent to $(1), i$. e., for all systems which could be obtained from (1) by a transformation of the form

$$
x=f(\bar{x}), \quad y=a(\bar{x}) \bar{y}+\beta(\bar{x}) \bar{z}, \quad z=\gamma(\bar{x}) \bar{y}+\delta(\bar{x}) \bar{z},
$$

which transformation was moreover the most general point-transformation capable of transforming a general system of form (1) into another of the same form and order. $f$

* Presented to the Society October 26, 1901. Received for publication October 4, 1901.

$\dagger$ E. J. WILCZYNSKI, Geometry of a simultaneous system of two linear homogeneous differential equations of the second order, Transactions of the American Mathematical Society, vol. 2 (1901), no. 4.

$\ddagger$ E. J. Wilczynski, Transformation of systems of linear differential equations, A merican Journal of Mathematics, vol. 23 (1901). 
Of course the following dualistic interpretation might also have been adopted. Let $\left(y_{k}\right)$ and $\left(z_{k}\right)$ be the coördinates of two planes, $p_{y}$ and $p_{z}$. Then equations (2) are the equations of two developable surfaces $D_{y}$ and $D_{z}$. Corresponding planes, $i$. e., planes corresponding to the same yalue of $x$, will intersect in a straight line $L_{y z}$, and the totality of such lines will generate a ruled surface $S$, which again cannot be a developable surface. If $x$ be left untransformed, the transformation (3) merely converts two planes $p_{y}$ and $p_{z}$ into two others $p_{\bar{y}}$ and $p_{\bar{z}}$ intersecting along the same line $L_{y z}$, while a transformation of $x$ interchanges these lines in an arbitrary manner. The transformation (3), then, does not change the ruled surface $S$, which is therefore characteristic of a class of systems of differential equations equivalent by a transformation of the form (3).

Let us combine the two interpretations. At corresponding points $P_{y}$ and $P_{z}$ of the two fundamental curves $C_{y}$ and $C_{z}$, let us construct the planes $p_{y}$ and $p_{z}$ tangent to the ruled surface $S$. These will intersect along the straight line $L_{y z}$ which joins $P_{y}$ and $P_{z}$. The four pairs of coördinates, determining these planes, will form a fundamental system of solutions for a new system of linear differential equations, as we shall show. This new system shall be called the adjoined of the original. Thus we shall have generalized the Lagrange equation adjoined to a single linear homogeneous differential equation.

\section{§2. Determination of the adjoined system.}

Let

so that

$$
D=\left|\begin{array}{cccc}
y_{1}^{\prime} & z_{1}^{\prime} & y_{1} & z_{1} \\
y_{2}^{\prime} & z_{2}^{\prime} & y_{2} & z_{2} \\
y_{3}^{\prime} & z_{3}^{\prime} & y_{3} & z_{3} \\
y_{4}^{\prime} & z_{4}^{\prime} & y_{4} & z_{4}
\end{array}\right|
$$

$$
D=\sum_{k=1}^{4} y_{k}^{\prime} v_{k}=-\sum_{k=1}^{4} z_{k}^{\prime} u_{k}
$$

where

$$
\begin{aligned}
& u_{1}=+\left(y_{2}^{\prime} y_{3} z_{4}\right), \quad u_{2}=-\left(y_{1}^{\prime} y_{3} z_{4}\right), \quad u_{3}=+\left(y_{1}^{\prime} y_{2} z_{4}\right), \quad u_{4}=-\left(y_{1}^{\prime} y_{2} z_{3}\right), \\
& v_{1}=+\left(z_{2}^{\prime} y_{3} z_{4}\right), \quad v_{2}=-\left(z_{1}^{\prime} y_{3} z_{4}\right), \quad v_{3}=+\left(z_{1}^{\prime} y_{2} z_{4}\right), \quad v_{4}=-\left(z_{1}^{\prime} y_{2} z_{3}\right),
\end{aligned}
$$

the symbol

$$
\left(a_{i} b_{j} c_{k}\right)
$$

denoting a determinant of the third order whose main diagonal is $a_{i} b_{j} c_{k}$.

It is evident that the homogeneous coördinates of the planes tangent to the integrating ruled surface of system $(1)$ at the points $\left(y_{i}\right)$ and $\left(z_{i}\right)$ respectively, are $\left(u_{i}\right)$ and $\left(v_{i}\right)$.

We shall now prove the following theorem: If the two fundamental curves 
$C_{y}$ and $C_{z}$, on the integrating ruled surface $S$, are transformed into two other curves $C_{\bar{y}}$ and $C_{\bar{z}}$ on the surface, by the transformation

$$
y_{k}=a(x) \bar{y}_{k}+\beta(x) \bar{z}_{k}, \quad z_{k}=\gamma(x) \bar{y}_{k}+\delta(x) \bar{z}_{k} \quad(k=1,2,3,4),
$$

the developable surfaces formed by the planes tangent to the surface $S$ along $C_{y}$ and $C_{z}$ are transformed by the equations

$$
u_{k}=\Delta\left[a(x) \bar{u}_{k}+\beta(x) \bar{v}_{k}\right], \quad v_{k}=\Delta\left[\gamma(x) \bar{u}_{k}+\delta(x) \bar{v}_{k}\right] \quad(k=1,2,3,4),
$$
where

$$
\Delta=a \delta-\beta \gamma,
$$

i. e., except for the factor $\Delta$, by cogredient transformations.

Proof. We have, the summation everywhere being for $k=1,2,3,4$,

$$
\begin{aligned}
& \sum u_{k} z_{k}^{\prime}=-D, \quad \sum u_{k} y_{k}^{\prime}=0, \quad \sum u_{k} z_{k}=0, \quad \sum u_{k} y_{k}=0, \\
& \sum v_{k} z_{k}^{\prime}=0, \quad \sum v_{k} y_{k}^{\prime}=D, \quad \sum v_{k} z_{k}=0, \quad \sum v_{k} y_{k}=0 .
\end{aligned}
$$

Moreover it is clear that these eight equations are just sufficient to determine $u_{k}$ and $v_{k}$. The values (7) of $y_{k}$ and $z_{k}$ being introduced, the following system, of which again $u_{k}, v_{k}$ are the unique solutions, is obtained:

$$
\begin{array}{lll}
\sum u_{k}\left(\gamma \bar{y}_{k}^{\prime}+\delta \bar{z}_{k}^{\prime}+\gamma^{\prime} \bar{y}_{k}+\delta^{\prime} \bar{z}_{k}\right)=-D, & \sum u_{k}\left(\gamma \bar{y}_{k}+\delta \bar{z}_{k}\right)=0, \\
\sum u_{k}\left(a \bar{y}_{k}^{\prime}+\beta \bar{z}_{k}^{\prime}+\alpha^{\prime} \bar{y}_{k}+\beta^{\prime} \bar{z}_{k}\right)=0, & & \sum u_{k}\left(a \bar{y}_{k}+\delta \bar{z}_{k}\right)=0, \\
\sum v_{k}\left(\gamma \bar{y}_{k}^{\prime}+\delta \bar{z}_{k}^{\prime}+\gamma^{\prime} \bar{y}_{k}+\delta^{\prime} \bar{z}_{k}\right)=0, & & \sum v_{k}\left(\gamma \bar{y}_{k}+\delta \bar{z}_{k}\right)=0, \\
\sum v_{k}\left(a \bar{y}_{k}^{\prime}+\beta \bar{z}_{k}^{\prime}+\alpha^{\prime} \bar{y}_{k}+\beta^{\prime} \bar{z}_{k}\right)=D, & \sum v_{k}\left(a \bar{y}_{k}+\beta \bar{z}_{k}\right)=0 .
\end{array}
$$

By direct computation we find

$$
D=\Delta^{2} \bar{D}
$$

Moreover we have the relations (9) between the transformed quantities $\bar{D}, \bar{u}_{k}$, $\bar{v}_{k}, \bar{y}_{k}, \bar{z}_{k}$, i. e.,

$$
\begin{aligned}
& \sum \bar{u}_{k} \bar{z}_{k}^{\prime}=-\bar{D}=-D_{l}^{\prime} \Delta^{2}, \quad \sum \bar{u}_{k} \bar{y}_{k}^{\prime}=0, \quad \sum \bar{u}_{k} \bar{z}_{k}=0, \quad \sum \bar{u}_{k} \bar{y}_{k}=0, \\
& \sum \bar{v}_{k} \bar{z}_{k}^{\prime}=0, \quad \sum \bar{v}_{k} \bar{y}_{k}^{\prime}=\bar{D}=D / \Delta^{2}, \quad \sum \bar{v}_{k} \bar{z}_{k}=0, \quad \sum \bar{v}_{k} \bar{y}_{k}=0 .
\end{aligned}
$$

Multiplying the first four equations of this set in order by $\delta, \gamma, \delta^{\prime}, \gamma^{\prime}$ and adding, we get the first equation of the following system :

$$
\begin{aligned}
& \sum \bar{u}_{k}\left(\gamma \bar{y}_{k}^{\prime}+\delta \bar{z}_{k}^{\prime}+\gamma^{\prime} \bar{y}_{k}+\delta^{\prime} \bar{z}_{k}\right)=-\delta D / \Delta^{2}, \quad \sum \bar{u}_{k}\left(\gamma \bar{y}_{k}+\delta \bar{z}_{k}\right)=0, \\
& \sum \bar{u}_{k}\left(a \bar{y}_{k}^{\prime}+\beta \bar{z}_{k}^{\prime}+a^{\prime} \bar{y}_{k}+\beta^{\prime} \bar{z}_{k}\right)=-\beta D / \Delta^{2}, \quad \sum \bar{u}_{k}\left(a \bar{y}_{k}+\beta \bar{z}_{k}\right)=0, \\
& \sum \bar{v}_{k}\left(\gamma \bar{y}_{k}^{\prime}+\delta \bar{z}_{k}^{\prime}+\gamma \bar{y}_{k}+\delta^{\prime} \bar{z}_{k}\right)=\gamma D_{j}^{\prime} \Delta^{2}, \quad \sum \bar{v}_{k}\left(\gamma \bar{y}_{k}+\delta \bar{z}_{k}\right)=0, \\
& \sum \bar{v}_{k}\left(a \bar{y}_{k}^{\prime}+\beta \bar{z}_{k}^{\prime}+a^{\prime} \bar{y}_{k}+\beta \bar{z}_{k}\right)=a D_{i}^{\prime} \Delta^{2}, \quad \sum \bar{v}_{k}\left(a \bar{y}_{k}+\beta \bar{z}_{k}\right)=0 \text {. }
\end{aligned}
$$


But from (13) follows very easily a system of precisely the same form as (10), only with

$$
\Delta\left(a \bar{u}_{k}+\beta \bar{v}_{k}\right) \text { and } \Delta\left(\gamma \bar{u}_{k}+\delta \bar{v}_{k}\right)
$$

in place of $u_{k}$ and $v_{k}$. But equations (10) were sufficient to determine $u_{k}$ and $v_{k}$ completely. Therefore we must have

$$
u_{k}=\Delta\left(a \bar{u}_{k}+\beta \bar{v}_{k}\right), \quad v_{k}=\Delta\left(\gamma \bar{u}_{k}+\delta \bar{v}_{k}\right) \quad(k=1,2,3,4),
$$

which proves the theorem.

If we put

$$
U_{k}=\frac{u_{k}}{\sqrt{D}}, \quad V_{k}=\frac{v_{k}}{\sqrt{D}} \quad(k=1,2,3,4)
$$

where the sign of the square root may be chosen at will, $\left(U_{k}, V_{k}\right)$ are absolutely cogredient with $\left(y_{k}, z_{k}\right)$.

For $x=$ const. we single out a generator of the ruled surface $S$ and we consider two points $P_{y}$ and $P_{z}$ upon it, together with the planes tangent to $S$ at these points. The transformation (7) then transforms $P_{y}$ and $P$, and their tangent planes into $\boldsymbol{P}_{\bar{y}}$ and $\boldsymbol{P}_{\bar{z}}$ and their tangent planes. The transformation (7) is now a linear transformation, and from the fact that the tangent planes are transformed by the cogredient transformations (8) follows the well-known theorem that the anharmonic ratio of four points on any generator is the same as the anharmonic ratio of the corresponding tangent planes.

If $y_{k}$ and $z_{k}$ form a fundamental system of solutions of equations (1) the determinant $D$ does not vanish, i. e., the ruled surface $S$ is a non-developable surface. If the corresponding determinant for $u_{k}$ and $v_{k}$ be formed, its value turns out to be $D^{3}$, and therefore also different from zero.

We can therefore regard $u_{k}$ and $v_{k}$ as constituting a simultaneous fundamental system of solutions of a pair of equations of the same form as (1). We proceed to set up this system of differential equations.

Denote the minors of $z_{k}$ and $y_{k}$ in $D$ by $\zeta_{k}$ and $\eta_{k}$ respectively, so that

$$
D=\sum_{k=1}^{4} y_{k} \eta_{k}=\sum_{k=1}^{4} z_{k} \zeta_{k}
$$

where

$$
\begin{aligned}
& \eta_{1}=+\left(y_{2}^{\prime} z_{3}^{\prime} z_{4}\right), \eta_{2}=-\left(y_{1}^{\prime} z_{3}^{\prime} z_{4}\right), \eta_{3}=+\left(y_{1}^{\prime} z_{2}^{\prime} z_{4}\right), \eta_{4}=-\left(y_{1}^{\prime} z_{2}^{\prime} z_{3}\right) \\
& \zeta_{1}=-\left(y_{2}^{\prime} z_{3}^{\prime} y_{4}\right), \zeta_{2}=+\left(y_{1}^{\prime} z_{3}^{\prime} y_{4}\right), \zeta_{3}=-\left(y_{1}^{\prime} z_{2}^{\prime} y_{4}\right), \zeta_{4}=+\left(y_{1}^{\prime} z_{2}^{\prime} y_{3}\right)
\end{aligned}
$$

Then making use of (1) we shall find

$$
\begin{aligned}
& u_{k}^{\prime}=\zeta_{k}-p_{11} u_{k}-p_{12} v_{k}, \\
& v_{k}^{\prime}=-\eta_{k}-p_{21} u_{k}-p_{22} v_{k}
\end{aligned} \quad(k=1,2,3,4),
$$


whence

$$
\begin{array}{r}
\begin{aligned}
u_{k}^{\prime \prime}=\zeta_{k}^{\prime}-p_{11} \zeta_{k}+p_{12} \eta_{k}+\left(p_{11}^{2}+p_{12} p_{21}-p_{11}^{\prime}\right) u_{k} \\
+
\end{aligned} \\
\begin{aligned}
\left.v_{k}^{\prime \prime}=-\eta_{k}^{\prime}-p_{21} \zeta_{k}+p_{22} \eta_{k}+\left\{\left(p_{11}+p_{22}\right) p_{21}-p_{22}\right) p_{12}-p_{12}^{\prime}\right\} u_{k} \\
+
\end{aligned} \\
\text { By differentiating (16) we find }
\end{array}
$$

and from (17)

$$
\begin{aligned}
& \eta_{k}^{\prime}=-\left(p_{11}+p_{22}\right) \eta_{k}+q_{11} v_{k}-q_{21} u_{k}, \\
& \zeta_{k}^{\prime}=-\left(p_{11}+p_{22}\right) \zeta_{k}+q_{12} v_{k}-q_{22} u_{k},
\end{aligned}
$$

$$
\begin{aligned}
& \eta_{k}=-v_{k}^{\prime}-p_{21} u_{k}-p_{22} v_{k}, \\
& \zeta_{k}=u_{k}^{\prime}+p_{11} u_{k}+p_{12} v_{k} .
\end{aligned}
$$

Substituting these values in (18) we find that $\left(u_{k}, v_{k}\right)$ are simultaneous solutions of the following system of equations:

$$
\begin{aligned}
u^{\prime \prime}+\left(2 p_{11}+p_{22}\right) u^{\prime}+p_{12} v^{\prime}+ & \left\{p_{11}^{\prime}+q_{22}+\left(p_{11}+p_{22}\right) p_{11}\right\} u \\
& +\left\{p_{12}^{\prime}-q_{12}+\left(p_{11}+p_{22}\right) p_{12}\right\} v=0, \\
v^{\prime \prime}+p_{21} u^{\prime}+\left(2 p_{22}+p_{11}\right) v^{\prime}+ & \left\{p_{21}^{\prime}-q_{21}+\left(p_{11}+p_{22}\right) p_{21}\right\} u \\
+ & +\left\{p_{22}^{\prime}+q_{11}+\left(p_{11}+p_{22}\right) p_{22}\right\} v=0 .
\end{aligned}
$$

Moreover $\left(u_{k}, v_{k}\right)$ form a simultaneous fundamental system of (19), since, as we have already seen, their determinant does not vanish.

We shall prefer, in general, to use another system, namely, that one whose solutions are the functions $U_{k}$ and $V_{k}$ as defined by equations (14). Remembering that

$$
D=C e^{-\int\left(p_{11}+p_{22}\right) d x},
$$

one sees that we can obtain this other system from (19) by making the transformation

$$
u=U e^{-1 / 2 \int\left(p_{11}+p_{22}\right) d x}, \quad v=V e^{-1 / 2 \iint\left(p_{11}+p_{22}\right) d x}
$$

The resulting system is

$$
\begin{aligned}
& U^{\prime \prime}+p_{11} U^{\prime}+p_{12} V^{\prime}+\left\{q_{11}+\frac{1}{4}\left(u_{11}-u_{22}\right)\right\} U+\left(q_{12}+\frac{1}{2} u_{12}\right) V=0 \\
& V^{\prime \prime}+p_{21} U^{\prime}+p_{22} V^{\prime}+\left(q_{21}+\frac{1}{2} u_{21}\right) U+\left\{q_{22}+\frac{1}{4}\left(u_{22}-u_{11}\right)\right\} V=0,
\end{aligned}
$$

where $u_{\mathrm{t} k}$ are the same as the quantities so denoted in a previous paper.*

* E. J. WILCZYNSKI, Invariants of systems of linear differential equations, Transaotions of the American Mathematical Society, vol. 2 (1901), no. 1, p. 6. 
A third form, which may be convenient, is obtained by putting

$$
u=\lambda e^{-\int\left(p_{11}+p_{22}\right) d x}, \quad v=\mu e^{-\int\left(p_{11}+p_{22}\right) d x}
$$

Its fundamental solutions are

$$
\lambda_{k}=\frac{u_{k}}{D}, \quad \mu_{k}=\frac{v_{k}}{D} \quad(k=1,2,3,4) .
$$

The equations which $\lambda$ and $\mu$ satisfy are

$$
\begin{aligned}
& \lambda^{\prime \prime}-p_{22} \lambda^{\prime}+p_{12} \mu^{\prime}-\left(p_{22}^{\prime}-q_{22}\right) \lambda+\left(p_{12}^{\prime}-q_{12}\right) \mu=0, \\
& \mu^{\prime \prime}+p_{21} \lambda^{\prime}-p_{11} \mu^{\prime}+\left(p_{21}^{\prime}-q_{21}\right) \lambda-\left(p_{11}^{\prime}-q_{11}\right) \mu=0 .
\end{aligned}
$$

We shall speak of system (21) as the system adjoined to (1). Of course (19) and (22) have essentially the same properties as (21). But the relation of (21) to (1) is somewhat simpler because its solutions are absolutely cogredient with the solutions (1) under transformation (3), while those of (19) and (22) are cogredient with (1) except for a factor.

\section{§3. Properties of adjoined systems. Reciprocity.}

The relation of adjoined systems to each other is a very close one. In the first place they have the same seminvariants and invariants.

For, if we form the quantities $u_{i k}, v_{i k}, w_{i k}$ for the system (21) and denote them by capital letters, we find

$$
U_{11}=u_{22}, \quad U_{12}=-u_{12}, \quad U_{21}=-u_{21}, \quad U_{22}=u_{11},
$$

and similarly for $V_{i k}$ and $W_{i k}$.

The relation between systems (1) and (21) is a reciprocal one, i. e., if of two systems the second is the adjoined of the first, then the first is also the adjoined of the second.

For let us denote the coefficients of (21) by $P_{i k}$ and $Q_{i k}$. Then

$$
\begin{array}{ll}
P_{i k}=p_{i k}, & \\
Q_{11}=q_{11}+\frac{1}{4}\left(u_{11}-u_{22}\right), & Q_{12}=q_{12}+\frac{1}{2} u_{12}, \\
Q_{22}=q_{22}+\frac{1}{4}\left(u_{22}-u_{11}\right), & Q_{21}=q_{21}+\frac{1}{2} u_{21} .
\end{array}
$$

But this gives, on account of (23),

$$
\begin{array}{ll}
p_{i k}=P_{i k}, & \\
q_{11}=Q_{11}+\frac{1}{4}\left(U_{11}-U_{22}\right), & q_{12}=Q_{12}+\frac{1}{2} U_{12}, \\
q_{22}=Q_{22}+\frac{1}{4}\left(U_{22}-U_{11}\right), & q_{21}=Q_{21}+\frac{1}{2} U_{21},
\end{array}
$$

Trans. Am. Math. Soc. 5 
i. e., $p_{i k}$ and $q_{i k}$ are formed from $P_{i k}$ and $Q_{i k}$ just as $P_{i k}$ and $Q_{i k}$ are formed from $p_{i k}$ and $q_{i k}$. This proves the reciprocity of the two systems.

From (24) it will be noted that the adjoined system coincides with the original, if and only if

$$
u_{11}-u_{22}=u_{12}=u_{21}=0,
$$

i. e., ${ }^{*}$ if and only if the integrating ruled surface is of the second order.

This may also be shown very simply in another way. Since $-u_{k}$ and $v_{k}$ are the minors of $z_{k}^{\prime}$ and $y_{k}^{\prime}$ respectively, in $D$, we have

$$
\sum_{i=1}^{4} U_{i} y_{i}=0, \quad \sum_{i=1}^{4} V_{i} z_{i}=0, \quad \sum_{i=1}^{4} U_{i} z_{i}=0, \quad \sum_{i=1}^{4} V_{i} y_{i}=0 .
$$

But if the differential equations for $U$ and $V$ are the same as those for $y$ and $z$, we must have

$$
U_{i}=\sum_{k=1}^{4} c_{i k} y_{k}, \quad V_{i}=\sum_{k=1}^{4} c_{i k} z_{k} \quad(k=1,2,3,4)
$$

which, substituted in the preceding equations, show that the curves $C_{y}$ and $C_{\text {s }}$ are situated on the same quadratic surface, and that the line joining the points $P_{y}$ and $P_{s}$ is a generator of this surface.

The equations (25) form an invariant system. If then the two systems (21) and (1) are not identical, but merely equivalent, these equations (25) must also be fulfilled. We can therefore say more generally: a system of two linear differential equations of the second order is equivalent to its adjoined system, if and only if its integrating ruled surface is of the second order.

If the original system has either the semi-canonical or the canonical form the same is true of the adjoined system.

From our definitions of the quantities involved we have the following relations :

$$
\begin{aligned}
& \sum z_{k}^{\prime} U_{k}=-\sqrt{\bar{D}}, \quad \sum y_{k}^{\prime} V_{k}=+\sqrt{\bar{D}}, \\
& \sum y_{k} U_{k}=0, \quad \sum y_{k} V_{k}=0 \text {, } \\
& \sum z_{k} U_{k}=0, \quad \sum z_{k} V_{k}=0, \\
& \sum y_{k}^{\prime} U_{k}=0, \quad \sum z_{k}^{\prime} V_{k}=0,
\end{aligned}
$$

where $D$ is defined by equation (4) or more briefly,

$$
D=D\left(y_{k}^{\prime}, z_{k}^{\prime}, y_{k}, z_{k}\right) \text {. }
$$

* E. J. WILCZYNSKI, Geometry of a simultaneous system of two linear homogeneous differential equations of the second order, Transactions of the American Mathematioal Society, vol. 2 (1901), no. 4, equation (61). 
It follows from the reciprocity of the two systems, and may also be verified directly by differentiation of (27) that

$$
\begin{array}{ll}
\sum V_{k}^{\prime} y_{k}=-\sqrt{D}, & \sum U_{k}^{\prime} z_{k}=+\sqrt{ } \bar{D}, \\
\sum U_{k} y_{k}=0, & \sum U_{k} z_{k}=0, \\
\sum V_{k} y_{k}=0, & \sum V_{k} z_{k}=0, \\
\sum U_{k}^{\prime} y_{k}=0, & \sum V_{k}^{\prime} z_{k}=0,
\end{array}
$$

where $D$ in the first place stands for

$$
D\left(U_{k}^{\prime}, V_{k}^{\prime}, U_{k}, V_{k}\right) ;
$$

but this is the same as (28), for

$$
D\left(U_{k}^{\prime}, V_{k}^{\prime}, U_{k}, V_{k}\right)=D\left(y_{k}^{\prime}, z_{k}^{\prime}, y_{k}, z_{k}\right) .
$$

Now let $y_{k}$ and $z_{k}$ be simultaneously transformed into

$$
\bar{y}_{k}=\sum_{i=1}^{4} c_{k i} y_{i}, \quad \bar{z}_{k}=\sum_{k=1}^{4} c_{k i} z_{i} \quad(k=1,2,3,4),
$$

where $c_{k i}$ are constants, whose determinant does not vanish. We can look upon such a transformation as a change of the tetrahedron of reference.

Equations (27) will be satisfied by the transformed quantities as well. Therefore

$$
\begin{array}{ll}
\sum_{i=1}^{4} z_{i}^{\prime} \sum_{k=1}^{4} \frac{c_{k i}}{\sqrt{ } \bar{C}} \overline{U_{k}}=-\sqrt{D}, & \sum_{i=1}^{4} y_{i}^{\prime} \sum_{k=1}^{4} \frac{c_{k i}}{\sqrt{ } \bar{C}} \bar{V}_{k}=+\sqrt{D}, \\
\sum_{i=1}^{4} y_{i} \sum_{k=1}^{4} \frac{c_{k i}}{\sqrt{ } \bar{C}} \bar{U}_{k}=0, & \sum_{i=1}^{4} y_{i} \sum_{k=1}^{4} \frac{c_{k i}}{\sqrt{ } \bar{C}} \bar{V}_{k}=0, \\
\sum_{i=1}^{4} z_{i} \sum_{k=1}^{4} \frac{c_{k i}}{\sqrt{ } \bar{C}} \bar{U}_{k}=0, & \sum_{i=1}^{4} z_{i} \sum_{k=1}^{4} \frac{c_{k i}}{\sqrt{ } \bar{C}} \bar{V}_{k}=0, \\
\sum_{i=1}^{4} y_{i}^{\prime} \sum_{k=1}^{4} \frac{c_{k i}}{\sqrt{ } \bar{C}} \bar{U}_{k}=0, & \sum_{i=1}^{4} z_{i}^{\prime} \sum_{k=1}^{4} \frac{c_{k i}}{\sqrt{C}} \bar{V}_{k}=0,
\end{array}
$$

where $C$ denotes the determinant of the transformation (31).

But these are of exactly the same form as (27), except that $U_{k}$ and $V_{k}$ have been replaced by

$$
\frac{1}{\sqrt{C}} \sum_{k=1}^{4} c_{k i} \bar{U}_{k}, \quad \frac{1}{\sqrt{ } \bar{C}} \sum_{k=1}^{4} c_{k i} \bar{V}_{k}
$$

respectively. But on the other hand equations (27) are sufficient to determine $U_{k}$ and $V_{k}$ as their solutions. 
Therefore we must have

$$
U_{i}=\frac{1}{\sqrt{C}} \sum_{k=1}^{4} c_{k i} \bar{U}_{k}, \quad V_{i}=\frac{1}{\sqrt{C}} \sum_{k=1}^{4} c_{k i} \overline{V_{k}} \quad(i=1,2,3,4),
$$

or, solving for $\bar{U}_{k}$ and $\bar{V}_{k}$,

$$
\bar{U}_{k}=\frac{1}{\sqrt{ } C} \sum_{i=1}^{4} C_{k i} U_{i}, \quad \bar{V}_{k}=\frac{1}{\sqrt{ } \bar{C}} \sum_{i=1}^{4} C_{k i} V_{i} \quad(k=1,2,3,4),
$$

where $C_{k i}$ is the minor of $c_{k i}$ in the determinant

$$
C=\left|c_{k i}\right| \text {. }
$$

The sign of the square root must be so chosen that, for positive values of $C$, $\sqrt{C}>0$. For, if (31) reduces to the identical substitution, (32) also must reduce to identity. The determinant of the substitution (32) is equal to $C$, as is that of (31).

We can state our result as follows: If the elements $y_{i}$ and $z_{i}$ of a simultaneous fundamental system of (1) are made to undergo cogredient linear substitutions with constant coefficients and non-vanishing determinants, the corresponding solutions of the adjoined system of differential equations also undergo mutually cogredient linear substitutions with constant coefficients and with the same determinant. The coefficients of the second set of substitutions are the minors of those of the first set in their determinant, divided by the square root of that determinant. In a slightly modified sense then, the quantities $\left(y_{k}, z_{k}\right)$ and $\left(U_{k}, V_{k}\right)$ are contragredient. The quantities $\left(y_{k}, z_{k}\right)$ and $\left(\lambda_{k}, \mu_{k}\right)=\left(u_{k} / D, v_{k} / D\right)$ are contragredient in the ordinary sense of the word.

Upon this theorem rests the simple relation between the monodromic groups and the transformation groups of reciprocal systems of differential equations.

We can complete the relations (27) in an interesting manner. We have

$$
\begin{aligned}
& y_{k}^{\prime \prime}+p_{11} y_{k}^{\prime}+p_{12} z_{k}^{\prime}+q_{11} y_{k}+q_{12} z_{k}=0 \\
& z_{k}^{\prime \prime}+p_{21} y_{k}^{\prime}+p_{22} z_{k}^{\prime}+q_{21} y_{k}+q_{22} z_{k}=0
\end{aligned}
$$

If we multiply these equations by $U_{k}$ and $V_{k}$ successively and add, taking into account the relations (27) we shall find

$$
\begin{aligned}
&-\sum_{k=1}^{4} y_{k}^{\prime} U_{k}^{\prime}= \sum_{k=1}^{4} y_{k}^{\prime \prime} U_{k}=+p_{12} \sqrt{D}, \\
& \sum_{k=1}^{4} y_{k}^{\prime \prime} V_{k}=-p_{11} \sqrt{D}, \\
& \sum_{k=1}^{4} z_{k}^{\prime \prime} U_{k}=+p_{22} \sqrt{ } \bar{D}, \\
&-\sum_{k=1}^{4} z_{k}^{\prime} V_{k}^{\prime}=\sum_{k=1}^{4} z_{k}^{\prime \prime} V_{k}=-p_{21} \sqrt{D} .
\end{aligned}
$$


Also, if (27) and (34) are used,

$$
\sum_{k=1}^{4} y_{k}^{\prime} V_{k}^{\prime}=\frac{D^{\prime}}{2 \sqrt{D}}+p_{11} \sqrt{ } \bar{D}, \quad \sum_{k=1}^{4} z_{k}^{\prime} U_{k}^{\prime}=-\frac{D^{\prime}}{2 \sqrt{D}}-p_{22} \sqrt{D}
$$

Finally, using (21) as we have just used (1) to deduce (34), we have

$$
\begin{aligned}
& \sum_{k=1}^{4} U_{k}^{\prime \prime} y_{k}=+p_{12} \sqrt{D}, \quad \sum_{k=1}^{4} U_{k}^{\prime \prime} z_{k}=-p_{11} \sqrt{D}, \\
& \sum_{k=1}^{4} V_{k}^{\prime \prime} y_{k}=+p_{22} \sqrt{D}, \quad \sum_{k=1}^{4} V_{k}^{\prime \prime} z_{k}=-p_{21} \sqrt{D},
\end{aligned}
$$

as one sees also from the reciprocity of (1) and (21).

I have shown in former papers that every system of form (1) can be reduced to a semi-canonical form characterized by the conditions $p_{i k}=0$ and also that the particular curves thus obtained on the integrating ruled surface are the curved asymptotic lines.

The above formulæ furnish a new proof for the latter theorem. As a matter of fact a simpler reduction is sufficient to determine the asymptotic lines. For, let the given system be transformed into another for which merely $p_{12}=p_{21}=0$, while $p_{11}$ and $p_{22}$ may be arbitrary. Then the integral curves $C_{y}$ and $C_{z}$ on $S$ will be such that

$$
\begin{aligned}
& \sum_{k=1}^{4} U_{k} y_{k}=0, \sum_{k=1}^{4} U_{k} y_{k}^{\prime}=0, \quad \sum_{k=1}^{4} U_{k} y_{k}^{\prime \prime}=0, \\
& \sum_{k=1}^{4} V_{k} z_{k}=0, \quad \sum_{k=1}^{4} V_{k} z_{k}^{\prime}=0, \quad \sum_{k=1}^{4} V_{k} z_{k}^{\prime \prime}=0,
\end{aligned}
$$

i. e., the plane tangent to $S$ at $\left(y_{1}, y_{2}, y_{3}, y_{4}\right)$ is the osculating plane of the curve $C_{y}$ at that point, and the plane tangent to $S$ at $\left(z_{1}, z_{2}, z_{3}, z_{4}\right)$ is the osculating plane of $C_{z}$ at that point. Therefore $C_{y}$ and $C_{z}$ are asymptotic lines of the surface.

If, then, in any system of form (1), $p_{12}=p_{21}=0$, its integral curves are asymptotic lines on its integrating ruled surface.

If a given system (1) has by a first transformation been converted into another for which $p_{12}=p_{21}=0$, the semi-canonical form, for which $p_{11}$ and $p_{22}$ also vanish, can be obtained very easily by putting

$$
y=\eta e^{-\frac{1}{2} \int p_{11} d x}, \quad z=\zeta e^{-\frac{1}{2} \int p_{22} d x} .
$$

Since such a transformation merely multiplies $y_{1}, \ldots, y_{4}$ by the same factor, and similarly $z_{1}, \cdots, z_{4}$, it does not affect the significance of these quantities as the homogeneous coördinates of corresponding points on two asymptotic lines. 
It may be noted that, analytically, our theory rests upon the consideration of certain minors of the determinant $D$. By the consideration of other minors other similar theories may be developed. One of these theories has been already touched upon in a previous paper,* namely, that of the differential equation satisfied by the six determinants of the second order in the matrix

$$
\left|\begin{array}{llll}
y_{1} & y_{2} & y_{3} & y_{4} \\
z_{1} & z_{2} & z_{3} & z_{4}
\end{array}\right|,
$$

i. e., by the six homogeneous line-coördinates of the generating straight line $L_{y z}$ of the ruled surface $S$.

All of the systems of differential equations thus associated with (1) are of interest in connection with their geometrical interpretations, which are obvious enough. It is clear that a general theory of non-developable ruled surfaces can be conveniently built up as the geometric equivalent of the theory of a system of differential equations of form (1). This I have pointed out before, and a number of detached theorems of such a theory of ruled surfaces have already appeared in my papers. A systematic theory of ruled surfaces on such a basis appears to be a desideratum; and this I shall attempt to furnish on some future occasion.

University of California, Berkeley, September 25, 1901.

* Transactions of the American Mathematical Society, vol. 2 (1901), no. 4, §3. 